\title{
Efecto del proceso de combustión sobre la eficiencia total de una central térmica de vapor
}

\section{Effect of combustion processes on the total efficiency of a steam thermal power plant}

\author{
JIMENEZ-GARCIA, Juan A. $\dagger^{*}$, DURAN-GARCIA, Maria D., TORRES-TOVAR, Rigoberto y
} GASPAR-SANCHEZ, Noé

Universidad Autónoma del Estado de México

ID $1^{\text {er }}$ Autor: Juan A., Jimenez-Garcia / ORC ID: 0000-0002-7303-1284, Researcher ID Thomson: G-2711-2018, CVU CONACYT ID: 241737

ID $1^{\text {er }}$ Coautor: Maria D., Duran-Garcia / ORC ID: 0000-0003-3584-4783, Researcher ID Thomson: J-9111-2013

ID $2^{\text {do }}$ Coautor: Rigoberto, Torres-Tovar / ORC ID: 0000-0001-8734-3839, CVU CONACYT ID: 353766

ID $3^{\text {er }}$ Coautor: Noé, Gaspar-Sanchez, / ORC ID: 0000-0000-4597-8865. CVU CONACYT ID: 232164

DOI: $10.35429 /$ JSI.2019.11.3.10.18

Recibido 04 de Junio, 2019; Aceptado 30 de Septiembre, 2019

\section{Resumen}

En la presente investigación se desarrollo un modelo de simulación del cálculo térmico del hogar del generador de vapor de una central termoeléctrica de vapor de 350 MW, que opera bajo un ciclo Rankine con recalentamiento y regeneración. Se realizó un análisis, modificando algunos parámetros de la combustión (flujo de aire y combustible) y se determinaron las temperaturas alcanzadas por los productos de la combustión a la salida del hogar del generador de vapor, empleando el método de sustituciones sucesivas, aplicadas a la metodología del cálculo térmico, con un criterio de paro del error relativo aproximado de $\varepsilon_{-}$ra $<$ $1 \times 10^{-8}$. Se encontró que las temperaturas alcanzadas por los gases de combustión a la salida del hogar del generador de vapor para los regímenes del $100 \%, 75 \%$, $50 \%$ y $25 \%$ son: $1492.4^{\circ} \mathrm{C}, 1379.3^{\circ} \mathrm{C}, 1238.0^{\circ} \mathrm{C}$ y 980.0 ${ }^{\circ} \mathrm{C}$, respectivamente. Respecto al efecto del dosado de aire sobre la eficiencia térmica del ciclo, se observa que ésta se incrementa ligeramente con el incremento del dosado. Siendo la eficiencia térmica máxima del ciclo del $35.8 \%$

Ciclo Rankine, Combustión, Eficiencia, Transferencia de calor

\begin{abstract}
In the present investigation, a simulation model of the thermal calculation of the steam generator's steam generator $350 \mathrm{MW}$, operating under a Rankine cycle with superheat and regeneration, was developed. An analysis was carried out, modifying some parameters of the combustion (air and fuel flow) and the temperatures reached by the products of the combustion at the exit of the steam generator's home were determined, using the method of successive substitutions, applied to the thermal calculation methodology, with a stopping criterion of the approximate relative error of $\varepsilon_{-}$ra $<1$ $\mathrm{x} 10-8$. It was found that the temperatures reached by the combustion gases at the steam generator's outlet for the $100 \%, 75 \%, 50 \%$ and $25 \%$ regimes are: $1492.4^{\circ} \mathrm{C}$, $1379.3^{\circ} \mathrm{C}, 1238.0^{\circ} \mathrm{C}$ and $980.0^{\circ} \mathrm{C}$, respectively. Regarding the effect of air dosing on the thermal efficiency of the cycle, it is observed that it increases slightly with the increase of the dosage. Being the maximum thermal efficiency of the cycle of $35.8 \%$
\end{abstract}

Rankine Cycle, Combustion, Efficiency, Heat transfer

Citación: JIMENEZ-GARCIA, Juan A., DURAN-GARCIA, Maria D., TORRES-TOVAR, Rigoberto y GASPARSANCHEZ, Noé. Efecto del proceso de combustión sobre la eficiencia total de una central térmica de vapor. Revista de Innovación Sistemática. 2019. 3-11: 10-18

\footnotetext{
*Correspondencia al Autor (Correo electrónico: juan.jimenez.uaem@gmail.com)

$\uparrow$ Investigador contribuyendo como primer Autor.
} 


\section{Introducción}

A nivel mundial, se emplean distintas tecnologías para la generación de energía eléctrica, en centrales térmicas se genera el $66.7 \%(40.8 \%$ en carboelectricas, $21.6 \%$ en centrales de ciclo combinado y $4.3 \%$ centrales que queman combustóleo), el $16.4 \%$ en hidroeléctricas, $10.6 \%$ en nucleoeléctricas, $2.1 \%$ con biocombustibles y $4.2 \%$ otras tecnologías (Solar, eólica, geo termoeléctricas y mareomotriz) (IEA, 2016).

Debido a las medidas de sustentabilidad y cambio climático, se promueve el uso de energías limpias para la generación de energía eléctrica, desincentivando el uso de combustibles fósiles. De 2014 a 2015 a nivel mundial, se redujo el uso de carbón mineral en $7.6 \%$, el combustóleo en $-10.6 \%$, fuentes nucleares $-0.4 \%$, paralelamente la generación hidroeléctrica se redujo en $-1.9 \%$, no obstante, otras tecnologías limpias incrementaron su aportación como la eólica en $+16 \%$, solar $+17.8 \%$, biocombustibles $+2.5 \%$, (IEA, 2016).

La generación bruta de energía eléctrica en México por tipo de tecnología en 2012 se constituida por $45.6 \%$ Ciclo combinado, $20.6 \%$ termoeléctricas convencionales, $12.9 \%$ carboelectricas, $12 \%$ hidroeléctricas, $3.4 \%$ Nucleoeléctrica, $2.7 \%$ otras fuentes (geotérmica, eólica y solar fotovoltaica), así como $2.4 \%$, Turbogas y $0.4 \%$ Combustión interna. (CFE, 2014a). En los últimos años, se ha observado que la tendencia nacional es instalar centrales de ciclo combinado debido a la alta disponibilidad del gas natural, así como la conversión de las centrales termoeléctricas convencionales ya existentes.

En 2014, la Comisión Federal de Electricidad (CFE), poseía un fuerte programa de conversión de centrales termoeléctricas convencionales a duales (dualidad para quemar combustóleo o gas natural en el generador de vapor), que son proyectos de oportunidad, debido a los precios fluctuantes de combustóleo y a la alta disponibilidad de gas natural a corto plazo, estudios han demostrado su factibilidad y rentabilidad económica (CFE, 2014b).
Sin embargo, existen cuestiones técnicas para la conversión que requieren total conocimiento de los regímenes de operación de las centrales termoeléctricas en cuestión, en especial sobre los procesos de combustión dado que una reconversón implica modificar los parámetros térmicos del proceso de combustión y que afecta las características de la flama y la temperatura de los gases en la cámara de combustión, lo que impacta sobre la transferencia de calor en las superficies intercambiadoras como lo son las paredes del hogar y espeficiamente los recalentadores de vapor.

Así pues, analizando el contexto antes mostrado, se observa la importancia de realizar una optimización sobre los sistemas convencionales de generación de energía eléctrica (Montiel et al, 2019), particularmente en las CT de Combustóleo, ya que estas aún representan un porcentaje importante de la generación total aun cuando se han implementado políticas para realizar la conversión de las centrales termoeléctricas a gas natural (CFE, 2012a).

\section{Caso de Estudio}

La central termoeléctrica analizada, cuenta con una potencia instalada de $700 \mathrm{MW}$, con dos unidades de $350 \mathrm{MW}$. Cada unidad con una potencia nominal de $332.5 \mathrm{MW}$, con generadores de vapor con capacidad de producción de vapor principal de $325.4 \mathrm{Kg} / \mathrm{s}$ a $541^{\circ} \mathrm{C}$ y $288 \mathrm{Kg} / \mathrm{s}$ de vapor recalentado a $541^{\circ} \mathrm{C}$. La presión nominal del domo del generador de vapor es de 183 Bar y la presión de vapor recalentado es de 40 Bar.

Se emplea combustóleo con una composición química de $83.64 \% \mathrm{C}, 11.3 \%$ Hidrogeno, $4.2 \%$ Azufre y $0.86 \%$ N+O. (CFE, 1987), se considera un ciclo Rankine con Recalentamiento y dos Regeneraciones para el análisis, cuyo diagrama simplificado y esquemático, se presenta en la figura 1. 


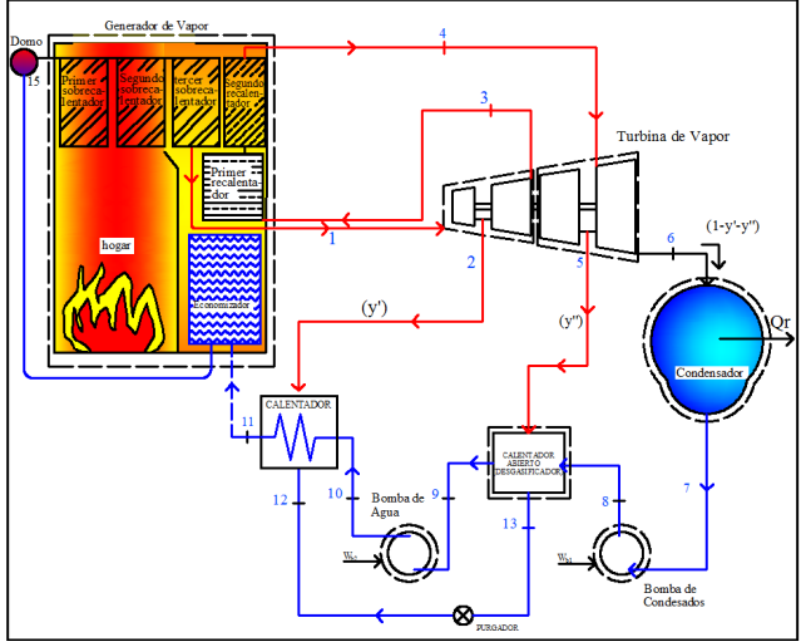

Figura 1 Diagrama Esquemático de la central termoeléctrica analizada

Fuente: Elaboración Propia

\section{Metodología del cálculo térmico de la cámara de combustión}

En el presente estudio se incorpora el análisis de proceso de la combustión en el hogar del generador de vapor, considerando variables independientes como el flujo de combustible, la relación aire- combustible y la fracción de gases de recirculación, en función de estas variables se determinan las propiedades de los gases de combustión como son su temperatura y flujo másico, lo que permitirá en los apartados siguientes realizar el análisis de la transferencia de calor.

La ecuación principal para el cálculo de intercambio de calor en hogares, conocida como ecuación de Gurvich (CKTI, 1973; Mullinger y Jenkins, 2007), se obtiene de la relación entre la temperatura relativa de los gases en salida del hogar $\left(\theta_{\text {hogar }}\right)$, el número de Boltzman $(B O)$, el número de Buger efectivo $(B \dot{u})$ y el parámetro $M$, que considera la distribución de temperaturas respecto a la altura del hogar y depende de la posición relativa del núcleo de la llama. Para su cálculo se utiliza la ecuación empírica que se define como (CKTI, 1998):

$\theta_{\text {hogar }}=\frac{T_{\text {hogar }}}{T_{a}}=\frac{B O^{0.6}}{M \cdot B U^{0.3+B O^{0.6}}}$

La temperatura $\theta_{\text {hogar }}$, es la relación entre la temperatura de los gases de salida del hogar $T_{\text {hogar }} \mathrm{y}$ la temperatura de flama adiabática de los productos de la combustión $T_{a}$
Alternativamente, la temperatura de los gases de combustión a la salida del hogar del generador de vapor analizado, se escribe como (CKTI, 1973):

$$
\begin{aligned}
& \mathrm{T}_{\text {hogar }}^{\prime \prime}= \\
& \frac{T_{a}}{1+M . B u^{0.3} *\left(\frac{5.67 . \Psi_{\text {promedio } * A_{\text {paredes }} * T_{a}^{3}}}{10^{11} \varphi B_{\text {calc }}(V C)_{\text {promedio }}}\right)^{0.6}}
\end{aligned}
$$

Donde, $\psi_{\text {promedio }}$ es el valor promedio del coeficiente de eficiencia térmica de las paredes del hogar y $A_{\text {paredes }}$ es el área de las paredes del hogar, $B_{\text {calc }}$ es el flujo de combustible suministrado a la cámara de combustión del generador de vapor, $\varphi$ es el coeficiente de conservación de calor en el hogar, $(V C)_{\text {promedio }}$ es el calor específico promedio de los productos de la combustión para $1 \mathrm{~kg}$ de combustóleo.

Las ecuaciones (2), permite también determinar la temperatura del núcleo de la llama $T_{a}$, el cual se puede considerar como un parámetro de diseño puesto que esta temperatura determina las propiedades termodinámicas de los gases de combustión.El cálculo de la temperatura de los gases de combustión a la salida del hogar y sus propiedades termodinámicas son necesarias para realizar el cálculo de la transferencia de calor en la zona de sobrecalentadores. Más información sobre la metodología se encuentra en Jiménez (2015).

Modelo de simulación del cálculo térmico del hogar del generador de vapor.

Para realizar el modelo de simulación del cálculo térmico del hogar del generador de vapor de $350 \mathrm{MW}$, se considera que el objetivo de esta simulación es conocer las propiedades termodinámicas de los gases de combustión a la salida del hogar (Calvo et al. 2019).

Para llevar a cabo el trabajo, se desarrolló una programación en macros de Excel en Visual Basic Applications, que permiten realizar los cálculos necesarios a fin de predecir las temperaturas de salida de los gases y vapor de agua en cada uno de los elementos del generador de vapor y del ciclo Rankine con recalentamiento y regeneración de la central termoeléctrica, que se muestra en la figura 2. 
Para simplificar el cálculo térmico del hogar y lograr una interface más amigable con el usuario, se creó un formulario, cuya caratula se puede apreciar en la figura 2 , en el que, se emplean las ecuaciones del calculo térmico de la cámara de combustión, con los que se determina la temperatura de los gases de combustión a la salida del hogar del generador de vapor.

Se emplea el método de aproximaciones sucesivas y se establece un criterio de paro, de un error relativo aproximado de $\varepsilon_{r a}<1 \times 10^{-8}$, entre iteraciones, lo que garantiza que el resultado tiene un error menor a $0.000000001 \%$.

El formulario de usuario de la figura 3, permite elegir diferentes configuraciones de las variables independientes como son: el exceso de aire en la mezcla aire - combustible, la fracción de gases de combustión que se recirculan al hogar del generador de vapor, cantidad de combustóleo que se inyecta a los quemadores del hogar e incluso la composición química del combustible.

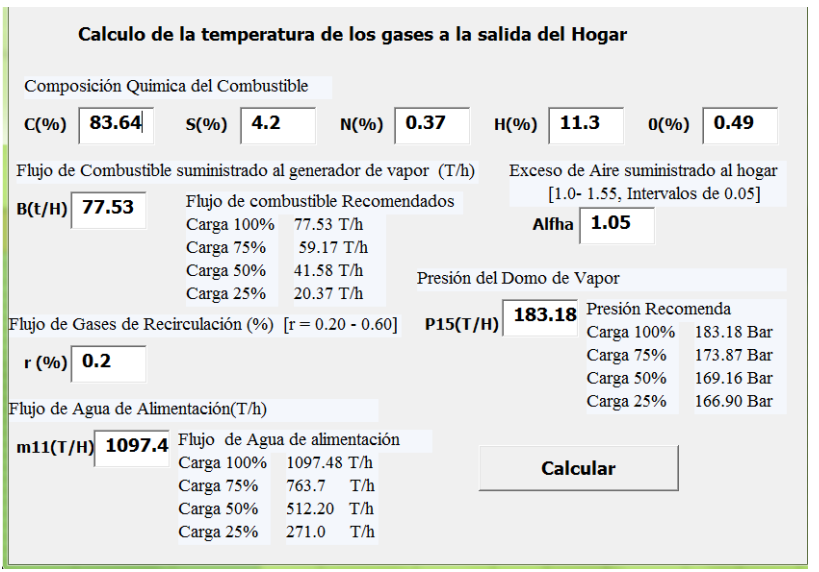

Figura 2 Formulario de usuario, para el cálculo térmico del hogar del generador de vapor de $350 \mathrm{MW}$

La central termoeléctrica Villa de Reyes trabaja en un ciclo cerrado, lo que propicia que exista una dependencia directa entre los cálculos térmicos, de transferencia de calor en los sobrecalentadores y el ciclo Rankine, por ello, para realizar la simulación del cálculo térmico del hogar, es necesario, definir los tipos de variables y las consideraciones tomadas, para tal propósito: a) Variables independientes, son aquellas variables de operación independientes, que se utilizan para iniciar la simulación del ciclo en su totalidad, entre ellas se encuentran: la presión del domo del generador de vapor, flujo másico de combustóleo, flujo másico de agua de alimentación y la fracción de gases de recirculación.

b) Variables semilla, en el cálculo térmico del hogar, es necesario considerar un valor semilla, para la temperatura adiabática de la flama en el hogar del generador de vapor, que por recomendaciones de la literatura es de 1323 K (Polupan, et al., 2002a), para realizar una primera iteración. Posteriormente los valores recalculados se ingresan de forma iterativa a la rutina programada a fin de encontrar su valor correspondiente. El diagrama de flujo del cálculo iterativo del cálculo térmico de la cámara de combustión se muestra en la figura 3.

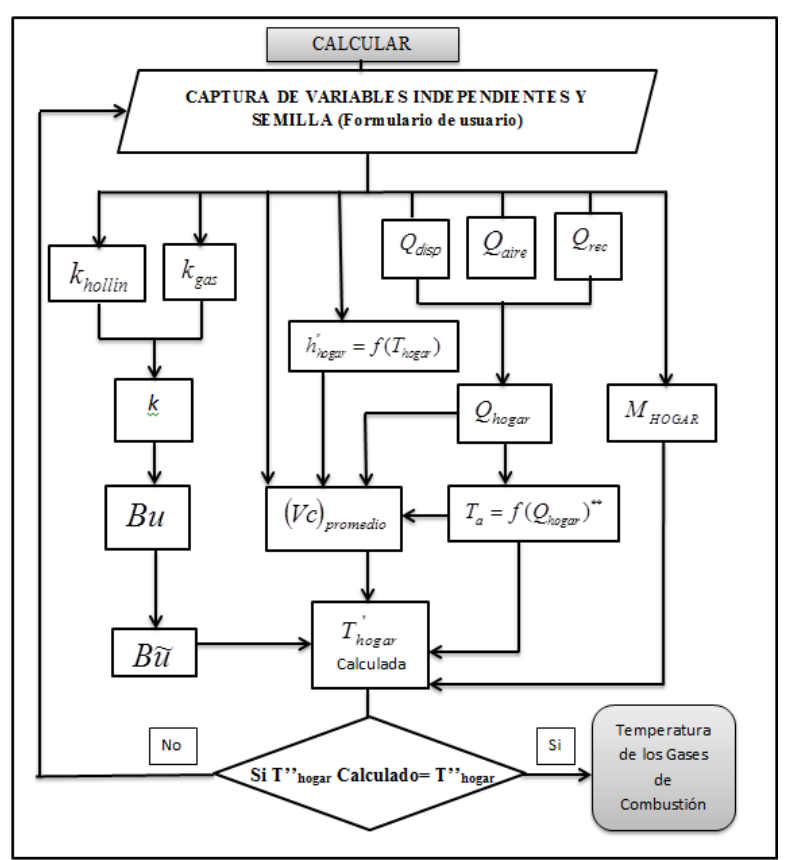

Figura 3 Diagrama de flujo del cálculo térmico del hogar del generador de vapor de $350 \mathrm{MW}$

Los resultados obtenidos del modelo termodinámico desarrollado se validan comparándolos directamente con los datos de operación proporcionados por el fabricante a los regímenes de carga de $100 \%, 75 \%, 50 \%$ y $25 \%$ (CFE, 1987). 
Asimismo, para realizar el cálculo térmico del hogar, se tomaron las siguientes consideraciones:

Se considera que el flujo de agua de alimentación es constante.

El agua de alimentación se suministra a una temperatura ambiente de $32^{\circ} \mathrm{C}$, según lo establece el manual de operación de la central termoeléctrica.

La composición química molar del combustible suministrado se considera de: $\mathrm{C}=83.64 \%, \quad \mathrm{~S}=4.2 \%, \quad \mathrm{~N}=0.37 \%$, $\mathrm{H}=11.3 \%, \mathrm{O}=0.49 \%$. Como lo establece el manual de operación de la central termoeléctrica, aunque el formulario de usuario (Ver figura 5) permite actualizar dicha composición química si fuera necesario

El combustóleo se suministra al hogar del generador de vapor a una temperatura de $135^{\circ}$ C. Según recomendaciones de operación de la central termoeléctrica.

Para lograr un combustión completa del combustóleo en el generador de vapor, se permite seleccionar en el formulario de usuario una inyección de exceso de aire mínimo del 5\% y máxima de $1.55 \%$

Para el presente estudio, se considera despreciable la caída de presión del lado de los gases de combustión. La caída de presión de lado del vapor se interpola en base a los parámetros establecidos en el MOGV para los regímenes de carga del $100 \%, 75 \%, 50 \%$ y $25 \%$, ya que en la presente investigación no se centra en el estudio hidráulico del sistema.

La geometría del hogar del generador de vapor y de los quemadores, para caracterizar el proceso de la combustión se considera constante y se toman con base en lo establecido en el manual de operación y construcción del generador de vapor.

$\mathrm{Se}$ establecen límites superiores e inferiores para los valores de las variables independientes en el ciclo térmico, esto es, se consideran siempre su valor, dentro del régimen máximo y mínimo establecido en el MOGV, para evitar condiciones peligrosas de operación.
El cálculo térmico del hogar se realizó a cargas parciales $(100 \%, 75 \%, 50 \%, 25 \%)$ con el fin de comparar los resultados calculados con los establecidos en el MOGV. La figura 4, muestra la temperatura de los gases de combustión a la salida del hogar, calculada con la ayuda del UserForm del cálculo térmico del hogar del generador de vapor de $350 \mathrm{MW}$.

Para realizar la simulación del cálculo térmico se emplearon los parámetros de la tabla 1 para los valores de las variables independientes.

\begin{tabular}{|l|l|l|l|l|}
\hline $\begin{array}{l}\text { Variable } \\
\text { independiente/Carga } \\
\text { Parcial }\end{array}$ & $\mathbf{1 0 0 \%}$ & $\mathbf{7 5 \%}$ & $\mathbf{5 0 \%}$ & $\mathbf{2 5 \%}$ \\
\hline $\begin{array}{l}\text { Flujo másico de } \\
\text { combustibles } \\
\left(\beta_{\text {comb }}\right)\end{array}$ & 77.53 & 59.17 & 41.58 & 20.37 \\
\hline Exceso de aire( $\alpha)$ & 1.05 & 1.05 & 1.30 & 1.15 \\
\hline $\begin{array}{l}\text { Fracción de } \\
\text { recirculación de } \\
\text { gases(r) }\end{array}$ & 0.20 & 0.30 & 0.40 & 0.35 \\
\hline $\begin{array}{l}\text { Agua de alimentación } \\
{[\mathrm{T} / \mathrm{h}]\left(\mathrm{m}_{\mathrm{a}}\right)}\end{array}$ & 1097.4 & 763.7 & 512.2 & 271 \\
\hline
\end{tabular}

Tabla 1 Variables independientes empeladas para el cálculo térmico del hogar a cargas parciales

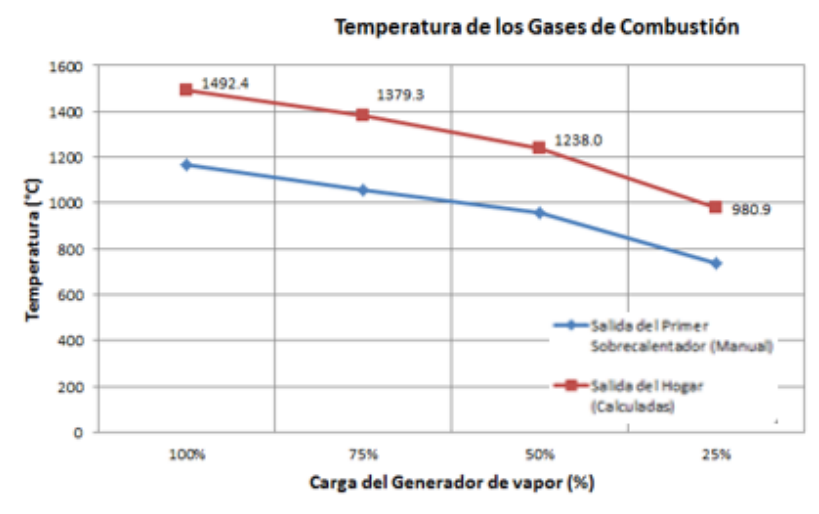

Figura 4 Tendencia de la temperatura de los gases a la salida del hogar, calculadas VS la temperatura de los gases a la salida del primer sobrecalentador, establecida en el MOGV

Por otra parte, en la figura 5 se muestra, la influencia que tiene el exceso de aire de la mezcla de aire-combustible, sobre la temperatura alcanzada por los gases de combustión. Podemos apreciar, que la mezcla con la que se presenta la mayor temperatura de los gases a la salida del hogar es con un $\alpha=1.10$, lo cual, concuerda con la literatura, que recomienda valores de aíre teórico entre $1.10 \mathrm{a}$ 1.15, para garantizar la quema del combustible en su totalidad. 
Se observa, que la máxima temperatura calculada para los gases de combustión es de $1503.2{ }^{\circ} \mathrm{C}$ con un exceso de aire de $\alpha=1.10$. Sin embargo, para el exceso de aire estequiométrico de $\alpha=1.0$, la temperatura de los gases de combustión es menor, siendo de $1497.6^{\circ} \mathrm{C}$, ya que en este régimen, parte del combustible no reacciona debido a la falta de aire en el proceso, lo que se conoce como combustión incompleta.

La temperatura de los gases de combustión disminuye conforme se aumenta el exceso de aire en el proceso de la combustión, esto, debido al aumento del volumen de los productos de la combustión en la zona de combustión activa del hogar del generador de vapor, que contribuye a distribuir la temperatura en el hogar, provocando temperaturas menores de los productos de la combustión a la salida del mismo.

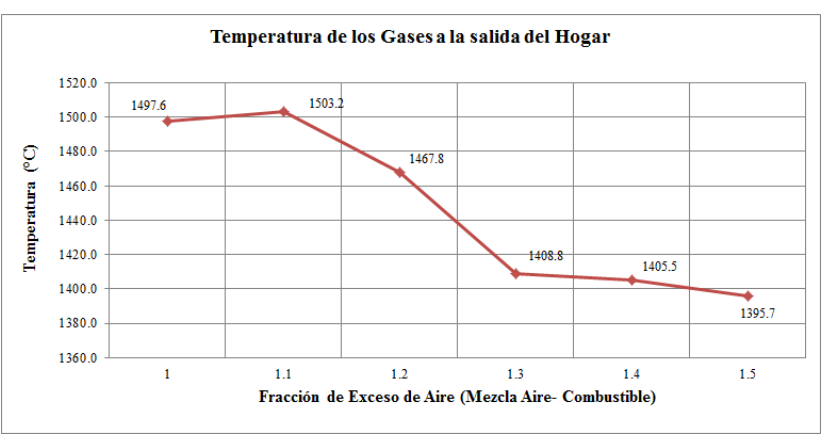

Figura 5 Temperatura de los gases a la salida del hogar del generador de vapor, para diferentes excesos de aire

Por otra parte, el mismo UserForm cálculo térmico del hogar (figura 5.3), permite también establecer la fracción de gases de combustión a la salida del economizador del generador de vapor, que se recirculan a la cámara de combustión, con la finalidad de aumentar la eficiencia térmica del generador. De manera particular en el MOGV, se especifican fracciones de recirculación que van desde el $20 \%$ hasta el $60 \%$, según el régimen de operación. Para las simulaciones realizadas en este apartado, se emplean las fracciones de recirculación establecidas en la tabla 1.

Es importante mencionar que el modelo del cálculo térmico, se ajusta adecuadamente a todos los regímenes de carga contras los que se comparan los resultados, por ello es factible emplear la metodología para cualquier régimen de carga diferente, ya que basta con modificar los valores de las variables independientes en el formulario (figura 2), para obtener resultados bajo condiciones de quemado diferentes.

\section{Efecto del Dosado de aire}

Para realizar el presente análisis, se consideran ocho diferentes valores de dosado de aire, desde el $10 \%$ de exceso de aire hasta el $40 \%$ de exceso de aire, así como el dosado establecido para la operación del generador de vapor a diferentes regímenes de operación. Se busca el dosado de aire optimo, que permita liberar toda la energía del combustible, a la vez de minimizan las pérdidas derivadas de las imperfecciones de la combustión y del exceso de aire. En la figura 6, se aprecia que el aumentar el dosado de aire en el proceso de la combustión, ocasiona que la temperatura de los productos de la combustión a la salida del hogar del generador de vapor (estado $T a$ ), disminuya. Esto debido al incremento en el flujo másico de gases de combustión, ya que existe mayor cantidad de aire.

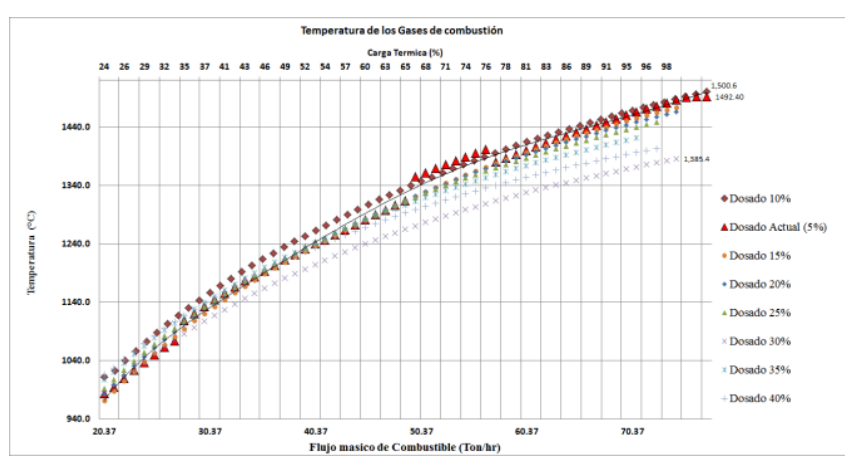

Figura 6 Temperatura de los gases de combustión a diferentes dosados de aire

Se encontró que la temperatura máxima calculada de los gases de combustión es de $1500.6^{\circ} \mathrm{C}$, con un dosado de aire del 1.1, que es $8.2^{\circ} \mathrm{C}$ superior a la temperatura de los gases de combustión calculada para el régimen actual de operación de la central termoeléctrica, que emplea para este régimen de carga, un dosado de 1.05. Al incrementar el exceso de aire al hogar del generador de vapor, la temperatura de los gases de combustión a la salida del hogar del generador de vapor decrece, hasta llegar a $1385.4^{\circ} \mathrm{C}$, cuando el dosado suministrado alcanza el 1.4 de exceso de aire y el generador de vapor opera al $100 \%$ de carga térmica.

Sin embargo, no siempre obtener la mayor temperatura de los gases de combustión a la salida del hogar del generador de vapor proporciona los mejores valores de eficiencia, ya que ésta es una combinación de las características del flujo de gases y vapor, así como de las temperaturas, ya que ambos participan en el proceso de intercambio de calor. 
Por lo que la eficiencia del generador de vapor muestra un incremento con el incremento del exceso de aire, ya que se mejora la turbulencia y la mezcla de los productos de la combustión en la zona de quemadores, lo que compensan la menor temperatura de los gases de combustión en el hogar del generador a carga parcial y permite mantener el rendimiento global del generador de vapor e incluso mejorarlo (Fernández, 2005).

En otras palabras, el incremento en el exceso de aire al hogar del generador de vapor, aun con la disminución en la temperatura de los gases de combustión a la salida del hogar del generador de vapor, es benéfico para el rendimiento térmico del generador de vapor, porque mejorar el proceso de transferencia de calor lo que se muestra en la figura 7 .

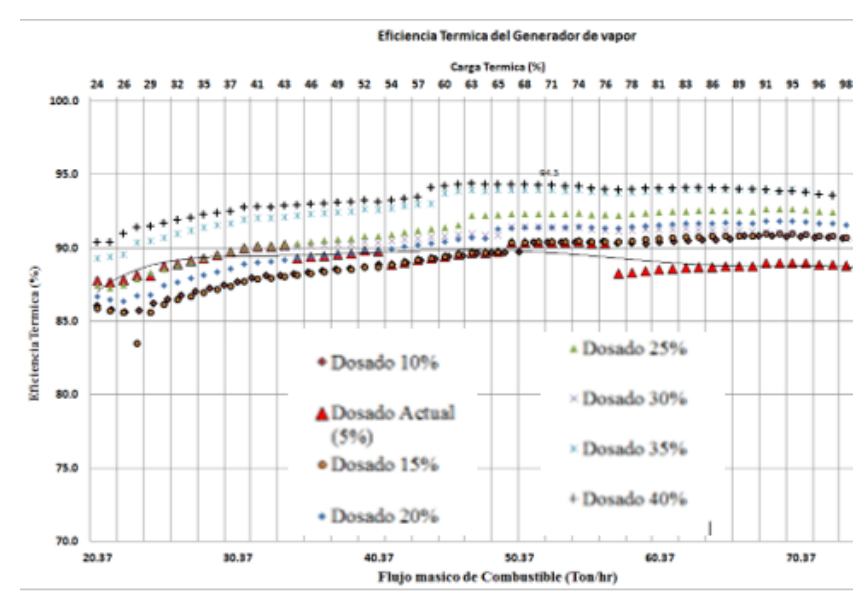

Figura 7 Eficiencia térmica del generador de vapor a diferentes dosados de aire

La eficiencia máxima del generador de vapor calculada para el dosado de aire del $40 \%$ es de $94.3 \%$ y se encuentra en el régimen de carga del $67 \%$, lo que se puede apreciar en la figura 6 .

\section{Conclusiones}

Después de desarrollar el modelo del cálculo térmico del hogar, donde se realizó un análisis, modificando algunos parámetros de la combustión se concluye que la metodología de cálculo térmico del hogar presentada en la presente investigación, predice adecuadamente la temperatura de los productos de la combustión a la salida del hogar del generador de vapor, empleando el métodos de sustituciones sucesivas con un criterio de paro del error relativo aproximado de $\varepsilon_{r a}<1 \times 10^{-8}$.
Con la metodología del cálculo térmico del hogar, se determinó que las temperaturas de los gases de combustión a la salida del hogar del generador de vapor para los regímenes del $100 \%, 75 \%, 50 \%$ y $25 \%$ son: $1492.4^{\circ} \mathrm{C}$, $1379.3^{\circ} \mathrm{C}, \quad 1238.0^{\circ} \mathrm{C} \quad$ y $\quad 980.0 \quad{ }^{\circ} \mathrm{C}$, respectivamente.

En la misma etapa, derivado del análisis del efecto del dosado de aire sobre la temperatura de los gases de combustión a la salida del hogar, se determinó que el dosado de aire que contribuye a producir la temperatura de los gases de combustión más alta es el de $\alpha$. $=1.10$. Siendo la máxima temperatura calculada de $1503.2^{\circ} \mathrm{C}$, para el régimen de carga del $100 \%$ y un dosado de $\alpha=1.10$.

Finalmente, en la etapa del análisis de sensibilidad se pudo concluir que el efecto del dosado de aire sobre el modelo del cálculo térmico del hogar y el ciclo de la central térmica que:

a. El incremento en el dosado de aire al hogar del generador de vapor ocasiona que la temperatura de los gases de combustión disminuya, y que el flujo de gases de combustión aumente. No obstante, el dosado de aire que produce la temperatura máxima de los gases de combustión es $\alpha=1.10$, siendo ésta de $1500.6^{\circ} \mathrm{C}$, a régimen de operación del $100 \%$ y con una eficiencia térmica de ciclo de $34.77 \%$. Sin embargo, con un dosado de aire de $\alpha=1.40$, se alcanza una eficiencia térmica máxima del ciclo de $35.55 \%$ a régimen de operación del $100 \%$, aun cuando la temperatura de los gases de combustión a la salida del hogar sea de $1385.4^{\circ} \mathrm{C}$. Con lo que se concluye, que el dosado de aire, afecta a la temperatura que alcanzan los gases de combustión, la cantidad de flujo másico de éstos y modifica las características físicas como turbulencia y velocidad, que afectan directamente a la transferencia de calor en los elementos subsecuentes.

b. Incrementar el dosado de aire hasta $\alpha=1.40$, incrementa la eficiencia térmica del generador de vapor. Siendo la eficiencia térmica máxima del $94.3 \%$, para un régimen de carga del $67 \%$. 
c. Respecto al efecto del dosado de aire sobre la eficiencia térmica del ciclo, se observa que ésta se incrementa ligeramente con el incremento del dosado. Siendo la eficiencia térmica máxima del ciclo del $35.8 \%$ al régimen de carga del $80 \%$. Sin embargo, se observa que el incremento en la eficiencia térmica del ciclo es más significativa debido al régimen de operación de la central, siendo los regímenes mayores al 75\%, lo que presentan mayores eficiencias térmicas.

d. Para el caso de la eficiencia exergética del ciclo, ésta se incrementa conforme lo hace el dosado de aire desde $\alpha=1.10$ hasta $\alpha=1.40$, para un mismo régimen de carga. La eficiencia exergética máxima calculada fue de $30.4 \%$ y se encontró en un régimen de operación del $66.6 \%$ con un dosado de aire de $\alpha=1.40$. La eficiencia exergética a partir del régimen de carga del $66.6 \%$, se comporta de una manera muy uniforme.

e. Con lo anterior, se concluye que, el incremento el en dosado de aire en la mezcla de aire combustible en el generador de vapor, contribuye a incrementar la eficiencia térmica del generador de vapor y las eficiencias térmica y exergética del ciclo. Sin embargo, como ya se dijo con anterioridad por cuestiones medioambientales el dosado de aíre debe de permanecer por debajo de $\alpha=1.20$. Por lo tanto, con base en los resultados obtenidos en la presente investigación se recomienda ampliamente emplear dosados de aire en el rengo del $\alpha=1.05$ al 1.15, que cumplen con la limitante medioambiental y permiten una buena combustión, así como, que con esta medida, se producen los gases de combustión a la salida del hogar del generador de vapor a mayor temperatura.

\section{Referencias}

Bejan, A. \& Kraus, A. (2003). Heat transfer Handbook, U.S.A.: John Wiley y Sons, Inc.
Calvo-González, A. E., Reyes-Becquer, N., Aballe-Infante, L., López-García, E., Domínguez-Machado, R., Santé-Heredia, V., \& Fernando-Mfumo, A. (2019). Cálculo térmico normativo de calderas digitalizado para el análisis de regímenes estacionarios. Ingeniería Mecánica, 22(2), 100-107.

Cengel, Y. A. \& Boles, M.A. (2012). Termodinámica (6 Ed). México: Mc Graw Hill.

CFE. (2012a). Works Program and Investment Electricity Sector 2012 - 2016 Mexico D.F: Programming Branch, Planning Coordination, CFE.

CFE. (2012b). Costs and Benchmarks for formulating investment projects Electricity Sector. México D.F.

CFE. (1987). Manual del Operación de la central termoeléctrica villa de reyes. San Luís Potosí, México: Comisión Federal de Electricidad.

CKTI. (1973). Thermal calculation of steam generator Regulatory Method (2 ed). Sankt Petersburg: CKTI.

CKTI. (1998). Thermal calculation of steam generator Regulatory Method (3 ed.) Sankt Petersburg: CKTI.

Duran M. (2004). Thermoeconomic optimization of combined cycle gas turbine using genetic algorithms, Tesis Doctoral, UPM, Madrid 2004.

Ebenhocha R., Matha D, Marathe S., Cortes P. \& Molins C. (2015). Comparative Levelized Cost of Energy Analysis. Energy Procedia 80 (2015), $108-122$.

Ganapathy, V., (1981). Estimate nonluminous radiation heat transfer coefficients. Hydrocarbon Processing, 235.

Ganapathy, V. (1982). Applied Heat Transfer. Tulsa OK: Penn Wll Book.

Habib, M. A., Said, S. A. \& Al-Zaharna, I. (1999). Thermodynamic optimization of reheat regenerative thermal-power plants. Applied Energy, 63. 
Jiménez, J. (2015). Análisis Exergético y Termoeconómico de un sistema de generación de energía, estudio del caso del generador de vapor de $305 \mathrm{MW}$ instalado en la central termoeléctrica Villa de Reyes. Tesis Doctoral. México. Universidad Autónoma del Estado de México.

Kitto, J., \& Stultz, S. (2005). Steam its generation and use (41 Edition), USA: The Babcock y Wilcox Company.

Kwak, H. Y., Kim, D. J., \& Jeon, J. S. (2003). Exergetic and thermoeconomic analyses of power plants. Energy, 28, pp. 343-360.

Montiel-Bohórquez, N. D., \& Pérez, J. F. (2019). Generación de Energía a partir de Residuos Sólidos Urbanos. Estrategias Termodinámicas para Optimizar el Desempeño de Centrales Térmicas. Información tecnológica, 30(1), 273284.

Moran M. (1997). Second Law application in thermal system design. In Boehm, R. (Ed.), Developments in the Design of thermal Systems. New York: Cambridge University Press.

Moran, M. \& Shapiro H. N. (2008). Fundamentals of engineering thermodynamics. (6th Ed.). USA: John \& Sons Inc.

Moran M. (2005). Engineering Thermodynamics. In: Kreith, F. \& Go swami D. Y., (Ed.). The CRC handbook of Mechanical Engineering.USA: The CRC press. Ch 2.

Mullinger P. \& Jenkins B. (2008). Industrial Process Furnaces, Principles and Design, Ed. Elsevier.

Narula, R. G. (1995). Salient design considerations for an ideal combined cycle power plant. Heat Recovery Systems y CHP, pp. 97-104.

Ndlovu, M. \& Majozi, T. (2014). Design and Optimization of Power Plant Utility Systems, Computer Aided Chemical Engineering, 34, 567-572.

Rovira A. (2004). Desarrollo de un Modelo para la Caracterización Termoeconómica de Ciclos Combinados de Turbinas de Gas y de Vapor en Condiciones de Carga Variable, Tesis Doctoral, UPM, Madrid, 2004.
SENER. (2017). Balance Nacional de Energía 2016. SENER. México.

Zhao, W., Zhang, T. \& Tang, X. (2012). Application of Power Electronic Technology in Optimizing the Operation of Thermal Power Plants. Advances in Mechanical and Electronic Engineering, 177. 